\title{
The importance of the excitation light polarization state for the optimization of the signal levels in two-photon light-sheet microscopy
}

Giuseppe de Vito $^{1,2}$, Pietro Ricci ${ }^{2}$, Lapo Turrini ${ }^{2,3}$, Vladislav Gavryusev $^{2,3}$, Caroline Müllenbroich ${ }^{2,4,5}$, Natascia Tiso 6 , Francesco Vanzi 2,7 , Ludovico Silvestri2,3,5, Francesco Saverio Pavone 2,3,5,8 ${ }^{1}$ University of Florence, Department of Neuroscience, Psychology, Drug Research and Child Health, Viale Pieraccini 6, Florence, FI 50139, Italy. ${ }^{2}$ European Laboratory for Non-Linear Spectroscopy, Via Nello Carrara 1, Sesto Fiorentino, FI 50019, Italy. ${ }^{3}$ University of Florence, Department of Physics and Astronomy, Via Sansone 1, Sesto Fiorentino, FI 50019, Italy. ${ }^{4}$ School of Physics and Astronomy, Kelvin Building, University of Glasgow, Glasgow, G12 8QQ, United Kingdom. ${ }^{5}$ National Institute of Optics, National Research Council, Via Nello Carrara 1, Sesto Fiorentino, FI 50019, Italy. ${ }^{6}$ University of Padova, Department of Biology, Via Ugo Bassi 58/B, Padua, PD 35131, Italy. ${ }^{7}$ University of Florence, Department of Biology, Via Madonna del Piano 6, Sesto Fiorentino, FI 50019, Italy.

8 francesco.pavone@unifi.it, Italy

\section{Abstract Text}

Two-photon (2P) light-sheet fluorescence microscopy (LSM) is a variant of traditional one-photon LSM that exploits the $2 \mathrm{P}$ absorption process to excite fluorescent dyes with infra-red light. Owing to the recent developments in scanning systems and fast cameras, in $2 \mathrm{P}$ LSM the volumetric acquisition frequency is limited by the signal-tonoise ratio and this is the reason why maximizing the signal levels is of paramount importance to achieve a high temporal resolution.

The polarization state of the excitation light plays an important, and 
often overlooked, role in the fluorescence excitation process. This is particularly true in 2P microscopy, due to the photoselection rules, and in LSM microscopy, due to the interplay between the directionality of the emitted fluorescent light and the orthogonality of the detection orientation; nevertheless, this role was not yet characterized for 2P LSM.

In this contribution, I will present our recent work [1] where we tested the observed 2P LSM signal levels for three different polarization states of the excitation light-circular polarization, linear polarization parallel to the detection axis, and linear polarization orthogonal to it-in five typical sample types characterized by spatially unordered dye populations: fluorescein solution and EGFPor GCaMP6s-expressing live and fixed zebrafish (Danio rerio) larvae.

In all observations, we consistently detected the highest level of signal for the linear polarization perpendicular to the detection axis, while the circular polarization produced lower signal levels. The linear polarization parallel to the detection axis produced high signal levels in the fluorescein solution and low signal levels in zebrafish larvae. These observations are in agreement with our theoretical predictions that link the (2P) LSM levels with the environmental fluorophore rotational mobilities.

In conclusion, our results highlight the importance of controlling the polarization state in (2P) LSM and show that, by carefully orienting the polarization axis of linearly-polarized light, it is possible to significantly increase (e.g. even more than doubling, for the living GCaMP6s case) the signal levels with respect to the orthogonallyoriented polarization, thus potentially enabling higher-speed 2P LSM [2]. 